\title{
Efecto de extractos de Sargassum spp. en el crecimiento y antioxidantes de plántulas de tomate
}

\section{Effect of extracts of Sargassum spp. in growth and antioxidants of tomato seedlings}

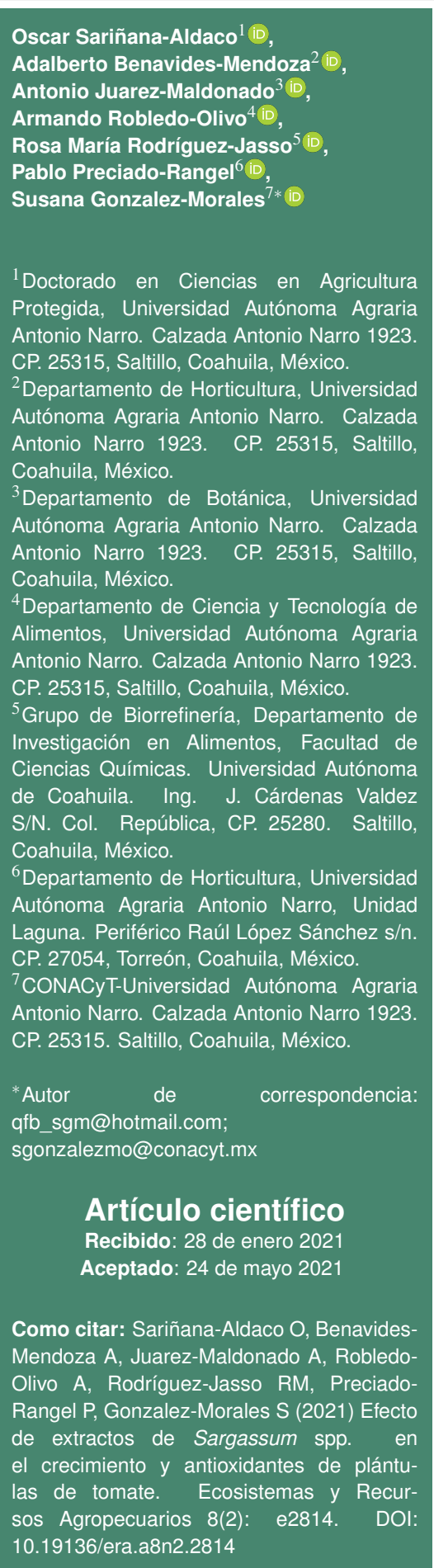

Oscar Sariñana-Aldaco

Adalberto Benavides-Mendoza

Antonio Juarez-Maldonado?

Armando Robledo-Olivo (D)

Rosa María Rodríguez-Jasso: 5

Pablo Preciado-Rangel 6 (D)

Susana Gonzalez-Morales [D

${ }^{1}$ Doctorado en Ciencias en Agricultura Protegida, Universidad Autónoma Agraria Coahuila, Mexico. Coahuila, México.

${ }^{3}$ Departamento de Botánica, Universidad Autónoma Agraria Antonio Narro. Calzada

${ }^{4}$ Departamento de Ciencia y Tecnología de Alimentos, Universidad Autónoma Agraria Ciencias Químicas. Universidad Autónoma de Coahuila. Ing. J. Cárdenas Valdez ${ }^{6}$ Departamento de Horticultura, Universidad Autónoma Agraria Antonio Narro, Unidad Periférico Raúl López Sánchez s/n. ahuila, México. Autonoma Agraria Antonio Narro. Calzada Antonio Narro 1923. Autor de afb_sgm@hotmail.com;

Artículo científico Recibido: 28 de enero 2021 Aceptado: 24 de mayo 2021

Como citar: Sariñana-Aldaco O, BenavidesMendoza A, Juarez-Maldonado A, RobledoOlivo A, Rodríguez-Jasso RM, PreciadoRangel P, Gonzalez-Morales S (2021) Efecto de extractos de Sargassum spp. 10.19136/era.a8n2.2814
RESUMEN. El tomate es la hortaliza más cultivada y consumida a nivel mundial y frecuentemente sufre diversas tensiones ambientales que afectan su productividad. Como posibles soluciones para mitigar el estrés está el uso de bioestimulantes, en particular, el uso de extractos de algas pardas que afectan positivamente varios procesos fisiológicos y bioquímicos en las plantas. Por lo tanto, el objetivo del presente trabajo fue evaluar el efecto de la aplicación foliar de 17 extractos hidroalcohólicos de Sargassum spp. al 1.5\%, más dos tratamientos control (agua destilada y un producto comercial a base de Ascophyllum nodosum) sobre el crecimiento y compuestos antioxidantes de plántulas de tomate, bajo un diseño completamente al azar. Los resultados muestran que el extracto que presentó los mejores resultados fue el EAS7 $\left(160{ }^{\circ} \mathrm{C} / 30 \mathrm{~min} / 50 \%\right.$ de etanol). Dicho extracto aumentó la altura, diámetro de tallo, materia fresca y seca total de las plántulas de tomate en un $8.67,32.32,25.03$ y $11.59 \%$, respectivamente, en comparación con el control absoluto (agua destilada). Además, el EAS7 aumentó el contenido de proteínas, glutatión, fenoles totales, flavonoides y capacidad antioxidante (ABTS) de las hojas en un 386.48, 15.92, 22.05, 35.99 y 18.86\%, respectivamente, en comparación con el control absoluto. La aplicación de extractos de Sargassum spp. es una alternativa prometedora para mejorar las características agronómicas y antioxidantes del cultivo de tomate.

Palabras clave: Bioestimulación, compuestos bioactivos, extractos de algas, Solanum lycopersicum L., sustentabilidad.

ABSTRACT. Tomato is the most cultivated and consumed vegetable in the world and is frequently attacked by various environmental stresses that affect its bioproductivity. Possible solutions to mitigate stress include the use of biostimulants, in particular the use of brown algae extracts that positively affect various physiological and biochemical processes in plants. Therefore, the objective of the present work was to evaluate the effect of foliar application of 17 hydroalcoholic extracts of Sargassum spp. at $1.5 \%$ and two control treatments (distilled water and commercial product based on Ascophyllum nodosum) on the growth and antioxidant compounds of tomato seedlings, under a completely random design. The results show that the extract called EAS7 $\left(160^{\circ} \mathrm{C} / 30 \mathrm{~min} / 50 \%\right.$ ethanol) presented the best results. EAS7 increased the height, stem diameter and total fresh and dry matter of tomato seedlings by $8.67,32.32,25.03$ and $11.59 \%$, respectively, compared to the absolute control (distilled water). Also, EAS7 increased the content of proteins, glutathione, total phenols, flavonoids and antioxidant capacity (ABTS) of the leaves by $386.48,15.92,22.05,35.99$ and $18.86 \%$, respectively, compared to the absolute control. The application of extracts of Sargassum spp. is a promising alternative to improve the agronomic and antioxidant characteristics of tomato crops.

Key words: Bioactive compounds, biostimulation, seaweed extracts, Solanum lycopersicum L., sustainability. 


\section{INTRODUCCIÓN}

El tomate es el cultivo hortícola más producido en el mundo, debido a que es una fuente natural de sustancias bioactivas de interés para la salud humana (Salehi et al. 2019). No obstante, constantemente es amenazado por diferentes tipos de estrés (salinidad, sequía, temperaturas extremas y patógenos), los cuales, reducen cantidad y calidad de los productos (Bai et al. 2018, González-García et al. 2019). El uso de bioestimulantes en la agricultura permite reducir el uso de agroquímicos ante situaciones de estrés, sin afectar de forma negativa el rendimiento de los cultivos o los ingresos de los agricultores (HernándezHerrera et al. 2014). Los bioestimulantes tienen la característica de inducir resistencia ante el estrés, mejorar la eficiencia en la absorción y asimilación de nutrientes y mejorar características agronómicas y de calidad de los cultivos (du Jardin 2015).

Los extractos de algas pardas tienen un alto poder bioestimulante, gracias a su contenido de vitaminas, carotenoides, fenoles, fitohormonas, proteínas, aminoácidos y azúcares, los cuales, al ser aplicados incrementan el rendimiento y la calidad en los cultivos (Ertani et al. 2018), además, inducen tolerancia al estrés (Jayaraman et al. 2011). Dichos extractos también son ricos en minerales como nitrógeno $(\mathrm{N})$ y potasio $(\mathrm{K})$ similares a los del estiércol vacuno y fertilizantes orgánicos, lo que los hace candidatos ideales para su uso en la agricultura (Trejo-Valencia et al. 2018). Además, hoy en día se encuentran disponibles una serie de extractos, los cuales, se pueden utilizar de manera foliar, en drench o en forma granular para acondicionar el suelo (Hernández-Herrera et al. 2014).

Las algas pardas del género Sargassum últimamente representan un problema turístico, ecológico y de salud pública, por las acumulaciones atípicas en las costas de México. Sin embargo, esta situación llevó a productores, comerciantes e investigadores a desarrollar diferentes productos a base de Sargassum, tales como: producción de combustibles, suplementos alimenticios y como bioestimulantes en el área agrícola (Brown et al. 2014, Pérez et al. 2018). Existen estudios que demuestran el poder bioestimulante de los productos derivados de Sargassum, como lo indican Kumari et al. (2011) quienes evaluaron el uso de extractos de Sargassum johnstonii en el cultivo del tomate, sus resultados indican mayor crecimiento del sistema radicular, incremento del número y peso de frutos, aumento de fenoles, licopeno y ácido ascórbico en los frutos.

El uso de extractos del alga Sargassum (EAS) es una alternativa para reducir el uso de químicos en la agricultura (N'Yeurt y Lese 2015), aumentar la tolerancia ante el estrés (Jayaraman et al. 2011) y mejorar el rendimiento y calidad de los cultivos (Abbas et al. 2020), además, representa una opción para mitigar el problema que causa esta alga en las costas. Por lo anterior, el objetivo del presente estudio fue determinar el efecto de la aplicación foliar de diferentes extractos hidroalcohólicos de Sargassum spp. sobre el crecimiento y los compuestos antioxidantes de plántulas de tomate.

\section{MATERIALES Y MÉTODOS}

\section{Producción de los extractos del alga Sargassum spp. (EAS)}

El alga Sargassum spp. fue recolectada en el mar Caribe mexicano. Las muestras se transportaron al laboratorio en recipientes de plástico y se lavaron con agua de grifo para eliminar las sales superficiales, luego se dejaron secar al aire libre el tiempo necesario hasta una humedad menor al $20 \%$. Después se procedió a la molienda hasta obtener un tamaño de partícula menor a $0.5 \mathrm{~mm}$. Posteriormente se llevó a cabo la extracción en la planta piloto del laboratorio de Biorrefinería de la Universidad Autónoma de Coahuila en Saltillo, México, donde se utilizó una relación 1:20 (1 g de alga y $20 \mathrm{~mL}$ de agua: etanol) (Ramya et al. 2015). La extracción se realizó en un reactor batch, siguiendo la metodología descrita por Ferreira et al. (2019) con modificaciones, considerando tres factores (temperatura, tiempo y porcentaje de etanol) y tres niveles por factor. Las condiciones de extracción se describen en la Tabla 1 , las cuales, se llevaron a cabo mediante un diseño central compuesto. Los extractos se filtraron a través de un papel filtro y se almacenaron a $-80{ }^{\circ} \mathrm{C}$ en un 
Tabla 1. Condiciones de extracción y caracterización de los EAS.

\begin{tabular}{ccccccc}
\hline $\begin{array}{c}\text { Tratamiento } \\
\text { EAS }\end{array}$ & $\begin{array}{c}\mathrm{T} \\
\left({ }^{\circ} \mathrm{C}\right)\end{array}$ & $\begin{array}{c}\text { TE } \\
(\mathrm{min})\end{array}$ & $\begin{array}{c}\text { Etanol } \\
(\%)\end{array}$ & $\begin{array}{c}\mathrm{PT} \\
\left(\mathrm{mg} \mathrm{g}^{-1} \mathrm{PF}\right)\end{array}$ & $\begin{array}{c}\mathrm{AA} \\
\left(\mathrm{mg} \mathrm{g}^{-1} \mathrm{PF}\right)\end{array}$ & $\begin{array}{c}\mathrm{GSH} \\
\left(\mathrm{mg} \mathrm{g}^{-1} \mathrm{PF}\right)\end{array}$ \\
\hline 1 & 120 & 0 & 0 & $1.30^{i}$ & $0.20^{j}$ & $1.78^{i}$ \\
2 & 120 & 0 & 50 & $0.91^{j}$ & $0.18^{k}$ & $1.61^{i}$ \\
3 & 120 & 30 & 50 & $1.90^{f}$ & $0.22^{i}$ & $2.37^{h}$ \\
4 & 120 & 30 & 0 & $1.63^{h}$ & $0.27^{h}$ & $2.87^{d e}$ \\
5 & 160 & 0 & 0 & $1.84^{f g}$ & $0.33^{e}$ & $3.09^{b-d}$ \\
6 & 160 & 0 & 50 & $2.18^{e}$ & $0.26^{h}$ & $2.59^{f-h}$ \\
7 & 160 & 30 & 50 & $3.47^{a}$ & $0.43^{a}$ & $3.35^{a b}$ \\
8 & 160 & 30 & 0 & $1.31^{i}$ & $0.33^{e}$ & $3.22^{a-c}$ \\
9 & 140 & 15 & 25 & $2.37^{b-d}$ & $0.33^{e}$ & $3.12^{a-d}$ \\
10 & 140 & 15 & 25 & $2.49^{b}$ & $0.34^{c d}$ & $3.14^{a-d}$ \\
11 & 140 & 15 & 25 & $2.45^{b c}$ & $0.34^{d}$ & $3.37^{a}$ \\
12 & 120 & 15 & 25 & $2.47^{b c}$ & $0.29^{g}$ & $2.76^{a-d}$ \\
13 & 160 & 15 & 25 & $2.39^{b c}$ & $0.35^{c}$ & $2.75^{a-d}$ \\
14 & 140 & 0 & 25 & $2.34^{c d}$ & $0.26^{h}$ & $2.53^{g h}$ \\
15 & 140 & 30 & 25 & $2.43^{b c}$ & $0.40^{b}$ & $3.01^{c-e}$ \\
16 & 140 & 15 & 0 & $1.75^{g h}$ & $0.29^{g}$ & $2.93^{d e}$ \\
17 & 140 & 15 & 50 & $2.23^{d e}$ & $0.30^{f}$ & $2.82^{e f}$ \\
\hline Letras diferentes dentro de cada columna indican diferencias significativas entre tratamien- \\
tos (LSD, p $\leq$ 0.05). EAS: Extracto de alga Sargassum spp.; T: Temperatura; TE: Tiempo \\
de extracción; PT: Proteínas totales; AA: Aminoácidos; GSH: Glutatión; PF: Peso fresco.
\end{tabular}

ultracongelador para su posterior análisis.

\section{Caracterización de los EAS}

A los 17 EAS producidos se les determinó la concentración de proteínas totales, aminoácidos y glutatión (Tabla 1). La cuantificación de proteínas totales se realizó mediante la técnica espectrofotométrica de Bradford (1976). En tubos de ensayo se agregaron $0.1 \mathrm{~mL}$ del EAS y $1 \mathrm{~mL}$ del reactivo Bradford para después dejar en reposo 5 minutos y leer en espectrofotómetro Uv-Vis (Genesis 10s UvVis, Thermo Scientific) a $595 \mathrm{~nm}$. Los aminoácidos se cuantificaron por la técnica propuesta por Yemm y Cocking (1955). En tubos de ensayo se agregaron $0.250 \mathrm{~mL}$ del EAS, $2.25 \mathrm{~mL}$ de buffer de citratos a $0.09 \mathrm{M}(\mathrm{pH} 5)$ y $0.5 \mathrm{~mL}$ de ninhidrina al $0.35 \%$, para luego agitarlos y colocarlos en baño María a temperatura de entre 80 y $100{ }^{\circ} \mathrm{C}$ durante $20 \mathrm{~min}$. Terminada la reacción, las muestras se colocaron en un baño de hielo y se leyó en un espectrofotómetro Uv-Vis (Genesis 10s Uv-Vis, Thermo Scientific) a $570 \mathrm{~nm}$. El glutatión se cuantificó siguiendo la metodología espectrofotométrica establecida por Xue et al. (2001), mediante la reacción del ácido 5,5 ditiobis-2 nitro benzoico (DTNB). En tubos de ensayo se agregaron $0.48 \mathrm{~mL}$ del EAS, $2.2 \mathrm{~mL}$ de $\mathrm{Na}_{2} \mathrm{HPO}_{4}$ a
$0.32 \mathrm{M}$ y $0.32 \mathrm{~mL}$ del colorante DTNB a $1 \mathrm{mM}$. Para luego mezclar y dejar reposar por 15 minutos para después realizar la lectura en un espectrofotómetro Uv-Vis (Genesis 10s Uv-Vis, Thermo Scientific) a 412 $\mathrm{nm}$. Los datos se reportaron en miligramos por gramo de peso fresco ( $\left.\mathrm{mg} \mathrm{g}^{-1} \mathrm{PF}\right)$.

\section{Material vegetal y condiciones experimentales}

Semillas de tomate hibrido el CID F1 de la compañía Harris Moran Seed Company, tipo saladette y crecimiento indeterminado, se sembraron en bandejas de poliestireno con una mezcla de sustrato de peat moss y perlita $(1: 1 \mathrm{v} / \mathrm{v})$. El experimento se estableció en un invernadero cubierto de polietileno en el Departamento de Horticultura de la Universidad Autónoma Agraria Antonio Narro en Saltillo, México. La temperatura promedio fue de 28 ${ }^{\circ} \mathrm{C}$ y 50 a $60 \%$ de humedad relativa. Las plántulas se cultivaron durante 30 días hasta que desarrollaron cuatro hojas verdaderas. Para luego trasplantarlas a contenedores de unicel de $0.5 \mathrm{~L}$ con la misma relación de sustrato utilizada en la siembra. El sistema de riego fue manual, dando un riego al día a capacidad de campo. La nutrición de las plantas se realizó con una solución nutritiva Steiner (1961) al $25 \%$. Las dosis aplicadas de los EAS fueron del 
$1.5 \%$ de acuerdo con investigaciones realizadas por Ramya et al. (2011), Kasim et al. (2015) y Ramya et al. (2015). Los EAS se aplicaron vía foliar utilizando un aspersor hasta el punto de goteo, las aplicaciones se realizaron cada 10 días a partir del trasplante, acumulando cuatro aplicaciones durante la prueba que tuvo una duración de 30 días a partir del trasplante. Las aplicaciones se realizaron entre las 08:00 y 10:00 h con un aspersor manual. Para evitar la pulverización de tratamientos vecinos, se utilizó una barrera plástica flexible para aislar el espacio de aplicación.

\section{Diseño experimental}

Se utilizó un diseño experimental completamente al azar con 19 tratamientos, los cuales fueron: 17 EAS y dos controles, un producto comercial de Ascophyllum nodosum y agua destilada como control absoluto. Cada tratamiento tuvo 10 réplicas, dando un total de 190 unidades experimentales.

\section{Variables evaluadas}

Se midieron las variables de crecimiento de altura de plántula, diámetro de tallo, número de hojas, peso fresco y peso seco total de las plántulas. Las variables bioquímicas evaluadas fueron: pigmentos fotosintéticos, proteínas totales, glutatión, fenoles totales, flavonoides y capacidad antioxidante hidrofílica (ABTS).

La altura de plántula se midió con un flexómetro desde la base de la superficie del suelo hasta el ápice de crecimiento. El diámetro del tallo con un vernier digital entre la primera y segunda hoja desde la base de la planta. También se contó el número de hojas y se tomó el peso freso y seco del vástago y raíz con una balanza digital.

Para determinar los compuestos bioquímicos en las plántulas de tomate, el material congelado se liofilizó. Para luego pulverizar la muestra en un mortero de porcelana. Para la extracción y cuantificación de proteínas, glutatión y capacidad antioxidante hidrofílica (ABTS) se colocaron $100 \mathrm{mg}$ de tejido liofilizado en un tubo de $2 \mathrm{~mL}$ y se agregaron $10 \mathrm{mg}$ de polivinilpirrolidona (PVP) para estabilizar los compuestos. Posteriormente se agregaron $2 \mathrm{~mL}$ de buffer de fosfatos $0.1 \mathrm{M}$ (pH 7-7.2), se homogeneizó en vórtex durante $20 \mathrm{~s}$, se sonicaron durante $5 \mathrm{~min}$ y se centrifugó a $12500 \mathrm{rpm} \mathrm{a} 4^{\circ} \mathrm{C}$ durante 10 minutos, para luego recolectar el sobrenadante para realizar el análisis. Con la extracción se realizó la cuantificación de proteínas y glutatión, con las metodologías descritas en al apartado de caracterización de EAS y los resultados se reportaron en miligramos por gramo de peso seco ( $\mathrm{mg} \mathrm{g}^{-1} \mathrm{PS}$ ).

La capacidad antioxidante hidrofílica (ABTS), se determinó por el método descrito por Re et al. (1999), que se basa en la decoloración del catión radical $A B T S$. El radical se obtuvo de la reacción del ABTS a $7 \mathrm{mM}$ con persulfato de potasio a $2.45 \mathrm{mM}$ $(1: 1 \mathrm{v} / \mathrm{v})$ en la oscuridad durante $16 \mathrm{~h}$, posteriormente se diluyó con etanol al $20 \%$ hasta obtener una absorbancia de $0.7 \pm 0.01$ a $754 \mathrm{~nm}$. Luego, para determinar la capacidad antioxidante de los compuestos hidrofílicos, en un tubo de $2 \mathrm{~mL}$ se colocaron $20 \mu \mathrm{L}$ del extracto y $980 \mu \mathrm{L}$ de la dilución del radical ABTS, se agitaron durante $5 \mathrm{~s}$ y se dejaron reposar $7 \mathrm{~min}$ en oscuridad. La absorbancia se medió en un espectrofotómetro Uv-Vis (Genesis 10s Uv-Vis, Thermo Scientific) a $754 \mathrm{~nm}$. Los resultados se expresaron en miligramos equivalentes de ácido ascórbico por gramo de peso seco (mg EAA g ${ }^{-1} \mathrm{PS}$ ).

Los pigmentos se determinaron con el método descrito por Wellburn (1994), con ligeras modificaciones, para lo cual, se agregaron $60 \mathrm{mg}$ de tejido liofilizado en un tubo de ensayo, para luego adicionar $5 \mathrm{~mL}$ de metanol puro e incubar a temperatura ambiente en oscuridad por $24 \mathrm{~h}$ para la extracción completa de los pigmentos. Pasadas las 24 h se procedió a medir la absorbancia en espectrofotómetro Uv-Vis (Genesis 10s Uv-Vis, Thermo Scientific) a 666, 653 y $470 \mathrm{~nm}$. La concentración de los pigmentos se expresa en miligramos por gramo de peso seco $\left(\mathrm{mg} \mathrm{g}^{-1}\right.$ PS), usando las siguientes formulas:

$$
\begin{gathered}
\text { Chl } a=[15.65(A 666)-7.34(A 653)] \\
\text { Chl } b=[27.05(A 653)-11.21(A 666)]
\end{gathered}
$$

Carotenoides $=\frac{[(1000 \mathrm{~A} 470)-2.86(\mathrm{Chl} \mathrm{a})-129.2(\mathrm{Chl} b)]}{221}$

Los fenoles se determinaron según la 
metodología de Singleton et al. (1999). La muestra se extrajo colocando $100 \mathrm{mg}$ de tejido liofilizado en un tubo de $2 \mathrm{~mL}$ y se agregaron $2 \mathrm{~mL}$ de solución de extracción (agua: acetona 1:1 v/v). La mezcla se agitó mediante un vórtex durante $20 \mathrm{~s}$ para después llevar a sonicación por 5 min. Finalmente, las muestras se centrifugaron a $12000 \mathrm{rpm}$ durante $10 \mathrm{~min}$ a $4{ }^{\circ} \mathrm{C}$ para obtener el sobrenadante y proceder a la cuantificación. La cuantificación se realizó en tubos de ensayo al agregar $50 \mu \mathrm{L}$ de cada muestra, 200 $\mu \mathrm{L}$ del reactivo Folin Ciocalteu al 100\%, $500 \mu \mathrm{L}$ de carbonato de sodio al $20 \%$ y $5 \mathrm{~mL}$ de agua destilada. La mezcla se dejó reposar durante 30 minutos a 45 ${ }^{\circ} \mathrm{C}$. Posteriormente la mezcla se leyó a una longitud de onda de $750 \mathrm{~nm}$ en un espectrofotómetro Uv-Vis (Genesis 10s Uv-Vis, Thermo Scientific, Waltham, MA, USA). Los resultados de fenoles se expresan en miligramos equivalentes de ácido gálico por gramo de peso seco (mg EAG g ${ }^{-1} \mathrm{PS}$ ).

Los flavonoides se cuantificaron siguiendo método de Zhishen et al. (1999). Los compuestos fueron extraídos colocando $100 \mathrm{mg}$ de tejido liofilizado en tubos de $2 \mathrm{~mL}$ y agregando $2 \mathrm{~mL}$ metanol al $80 \%$. La mezcla se pasó por el vórtex durante $20 \mathrm{~s}$, posteriormente se sónico por 5 minutos y se centrifugo a $4000 \mathrm{rpm}$ durante $10 \mathrm{~min}$ a $4{ }^{\circ} \mathrm{C}$. Para la mezcla se colocaron $250 \mu \mathrm{L}$ de la alícuota en un tubo de ensayo, seguido de la adición de 75 $\mu \mathrm{L}$ de $\mathrm{NaNO}_{2}$ al $5 \%$ y se agitó mediante un vórtex. Después de 5 min se agregaron $150 \mu \mathrm{L}$ de $\mathrm{AlCl}_{3}$ al $10 \%$; luego, se agregó un volumen de $500 \mu \mathrm{L}$ de $\mathrm{NaOH} 1 \mathrm{M}$, más un volumen final de $2.025 \mathrm{~mL}$ de agua destilada. La absorbancia se midió a $510 \mathrm{~nm}$ en un espectrofotómetro Uv-Vis (Genesis 10s Uv-Vis). Los flavonoides se cuantificaron mediante una curva estándar de catequina, basados en peso seco (mg $\left.E C g^{-1} P S\right)$.

\section{Muestreo de plantas}

Para las variables de crecimiento se seleccionaron cinco plantas al azar y de la misma manera para compuestos antioxidantes. Se muestreó la hoja más joven totalmente expandida, la cual, se colocó en aluminio, se congeló inmediatamente con nitrógeno líquido y se almacenó en un ultraconge- lador a $-80{ }^{\circ} \mathrm{C}$ para su posterior análisis. El muestreo y evaluación de las plántulas se realizó 24 h después de la última aplicación de los EAS.

\section{Análisis de datos}

Se realizó un análisis de varianza y prueba de medias de la diferencia mínima significativa (LSD) de Fisher $(\mathrm{p} \leq 0.05)$. Todos los procedimientos estadísticos se realizaron utilizando el software Infostat (v2018)

\section{RESULTADOS}

\section{Caracterización de los EAS}

Los resultados indican que las diferentes condiciones de extracción causaron diferencias en la concentración de metabolitos nitrogenados en los EAS (Tabla 1). Se puede apreciar que el EAS que presentó mayores concentraciones de estos metabolitos es el EAS7 $\left(160{ }^{\circ} \mathrm{C} / 30 \mathrm{~min} / 50 \%\right.$ de etanol), el cual, muestra valores de $3.47,0.43$ y 3.35 $\mathrm{mg} \mathrm{g}^{-1}$ PF para proteínas, aminoácidos y glutatión, respectivamente. En cuanto al tratamiento que presentó los valores más bajos, fue el EAS2 $\left(120^{\circ} \mathrm{C} / 0\right.$ $\mathrm{min} / 50 \%$ etanol), dicho EAS muestra valores de 0.91 , 0.18 y $1.61 \mathrm{mg} \mathrm{g}^{-1}$ PF para proteínas, aminoácidos y glutatión, respectivamente. El resto de los EAS muestra valores entre los rangos del EAS2 y EAS7. De igual manera se puede apreciar que conforme aumenta la condición de extracción, la concentración de metabolitos en los EAS es mayor.

\section{Parámetros de crecimiento de las plántulas}

Los resultados señalan que las aplicaciones foliares de los EAS en las plántulas de tomate causaron cambios significativos $(p \leq 0.05)$ en los parámetros de crecimiento (Tabla 2 y 3 ). EI EAS con mejores resultados fue el denominado EAS7, este EAS aumentó la altura de plántula, número de hojas, diámetro de tallo, materia fresca y seca total en un $8.67,18.42$, $32.32,25.03$ y $11.59 \%$, respectivamente, en comparación con el control absoluto. En cuanto a la aplicación del producto comercial se observó un incremento en todas las variables en comparación al control absoluto, pero no superior que el de la mayoría de 
los EAS. Se puede apreciar que el control absoluto en todos los parámetros de crecimiento estadísticamente es de los más bajos, a excepción de la variable de altura de plántula, en donde el tratamiento más bajo es el EAS3 $\left(120^{\circ} \mathrm{C} / 30 \mathrm{~min} / 50 \%\right.$ etanol), dicho tratamiento fue superado por el control absoluto en un $7.59 \%$.

Tabla 2. Efecto de los EAS sobre variables de altura, número de hojas y diámetro de tallo en plántulas de tomate.

\begin{tabular}{cccc}
\hline $\begin{array}{c}\text { Tratamiento } \\
\text { (EAS) }\end{array}$ & $\begin{array}{c}\text { Altura } \\
(\mathrm{cm})\end{array}$ & $\begin{array}{c}\text { Número de } \\
\text { hojas }\end{array}$ & $\begin{array}{c}\text { Diámetro de tallo } \\
(\mathrm{mm})\end{array}$ \\
\hline 1 & $42.02^{c-f}$ & $8.2^{b-d}$ & $6.64^{c-f}$ \\
2 & $42.42^{b-f}$ & $7.8^{c d}$ & $6.24^{f g}$ \\
3 & $38.16^{g}$ & $8.4^{a-d}$ & $6.98^{b c}$ \\
4 & $41.92^{d-f}$ & $9.2^{a}$ & $77^{b c}$ \\
5 & $43.15^{a-f}$ & $8.6^{a-c}$ & $6.34^{a-d}$ \\
6 & $43.14^{a-f}$ & $8.4^{a-d}$ & $6.68^{c-e}$ \\
7 & $44.62^{a b}$ & $9^{a b}$ & $7.86^{a}$ \\
8 & $42.12^{c-f}$ & $8.8^{a b}$ & $7^{b c}$ \\
9 & $41.78^{e f}$ & $8.6^{a-c}$ & $6.52^{d-f}$ \\
10 & $43.64^{a-e}$ & $8.6^{a-c}$ & $6.5^{d-f}$ \\
11 & $43.38^{a-f}$ & $7.8^{c d}$ & $6.44^{d-f}$ \\
12 & $45.24^{a}$ & $8.4^{a-d}$ & $6.66^{c-f}$ \\
13 & $44.98^{a}$ & $9^{a b}$ & $7.02^{b c}$ \\
14 & $44.7^{a b}$ & $8.8^{a b}$ & $7.18^{b}$ \\
15 & $42.36^{b-f}$ & $9.2^{a}$ & $6.72^{c-e}$ \\
16 & $44.4^{a-c}$ & $8.4^{a-d}$ & $7.02^{b c}$ \\
17 & $44.3^{a-d}$ & $8.4^{a-d}$ & $6.84^{b-d}$ \\
PC & $43.82^{a-e}$ & $8.6^{a-c}$ & $6.44^{d-f}$ \\
T0 & $41.06^{f}$ & $7.6^{d}$ & $5.94^{g}$ \\
CV (\%) & 4.51 & 9.29 & 5.09 \\
\hline
\end{tabular}

Letras diferentes dentro de cada columna indican diferencias significativas entre tratamientos (LSD, $p \leq 0.05)$. EAS: Extracto de alga Sargassum spp.; PC: Producto comercial; T0: Control absoluto; CV: Coeficiente de variación.

\section{Pigmentos fotosintéticos}

La concentración de pigmentos en las hojas de las plántulas de tomate se incrementó sustancialmente $(p \leq 0.05)$ con la adición foliar de los EAS. Se puede apreciar que el mejor tratamiento, fue el EAS12 $\left(120^{\circ} \mathrm{C} / 15 \mathrm{~min} / 25 \%\right.$ etanol), el cual, incrementó la clorofila a, clorofila b, clorofila total y carotenoides en un 33.69, 25.91, 31.17 y 100\%, respectivamente, en comparación con el control absoluto, el cual, fue el que presentó los valores más bajos (Tabla 4). En cuanto al producto comercial, muestra valores intermedios de concentración.

\section{Proteínas y estado antioxidante de las plántulas}

En cuanto a las proteínas y compuestos antioxidantes de las plántulas de tomate el análisis estadístico muestra que se presentaron cambios significativos $(p \leq 0.05)$ por la adición foliar de los EAS. En cuanto al mejor tratamiento, fue el denominado EAS7, ya que, aumentó las proteínas, glutatión, fenoles totales, flavonoides (Figura 1) y la capacidad antioxidante hidrofílica ABTS (Figura 2) de las hojas en un $386.48,15.92,22.05,35.99$ y $18.86 \%$, respectivamente, en comparación al control absoluto, el cual, fue de los tratamientos que presentó los valores más bajos. En cuanto al producto comercial, presentó valores intermedios de concentración. En general el EAS7 fue superior al resto de los tratamientos ya que los superó estadísticamente en proteínas, glutatión, fenoles totales y flavonoides, pero no en capacidad antioxidante. En esta variable los mejores tratamientos fueron el EAS4 $\left(120{ }^{\circ} \mathrm{C} / 30 \mathrm{~min} / 0 \%\right.$ etanol) y EAS16 $\left(140{ }^{\circ} \mathrm{C} / 15 \mathrm{~min} / 0 \%\right.$ etanol), seguidos por el EAS7. Probablemente estos tratamientos son ricos en otros compuestos antioxidantes que no se determinaron, a lo cual, se debe su mayor capacidad antioxidante.

\section{DISCUSIÓN}

\section{Caracterización de los EAS}

Los extractos de algas pardas están ganando interés creciente, por su composición única de compuestos de interés en la agricultura. Recientemente se están utilizando diversas técnicas de extracción de compuestos biológicamente activos que son amables con el medio ambiente (Michalak y Chojnacka 2015, Shukla et al. 2019). Cada técnica de extracción que se utilice mostrará resultados diferentes en cuanto a la concentración de los compuestos de interés, por lo cual, dichas técnicas se tienen que elegir de acuerdo a los objetivos del estudio y a los materiales disponibles (Michalak y Chojnacka 2014). En este estudio la extracción se realizó mediante un reactor batch controlando temperaturas, tiempos y porcentaje de etanol. Por lo que los resultados obtenidos en la caracterización de los EAS se pueden atribuir las condiciones a las que fue sometida el alga, lo que 
Tabla 3. Efecto de los EAS sobre biomasa aérea y de raíz en plántulas de tomate.

\begin{tabular}{ccccccc}
\hline $\begin{array}{c}\text { Tratamiento } \\
\text { (EAS) }\end{array}$ & $\begin{array}{c}\text { PFV } \\
(\mathrm{g})\end{array}$ & $\begin{array}{c}\text { PFR } \\
(\mathrm{g})\end{array}$ & $\begin{array}{c}\text { PSV } \\
(\mathrm{g})\end{array}$ & $\begin{array}{c}\text { PSR } \\
(\mathrm{g})\end{array}$ & $\begin{array}{c}\text { PFT } \\
(\mathrm{g})\end{array}$ & $\begin{array}{c}\text { PST } \\
(\mathrm{g})\end{array}$ \\
\hline 1 & $53.06^{d}$ & $7.43^{b-e}$ & $16.04^{d-f}$ & $2.09^{c-f}$ & $60.49^{d-f}$ & $18.13^{e-h}$ \\
2 & $52.44^{d}$ & $5.95^{f-h}$ & $16.08^{c-f}$ & $1.59^{g h}$ & $58.38^{f}$ & $17.67^{f-h}$ \\
3 & $53.91^{d}$ & $9.37^{a}$ & $16.20^{b-f}$ & $2.55^{a b}$ & $63.27^{c-f}$ & $18.75^{b-e}$ \\
4 & $57.31^{b-d}$ & $7.51^{b-e}$ & $16.53^{a-e}$ & $2.08^{c-f}$ & $64.82^{c-e}$ & $18.61^{c-e}$ \\
5 & $52.83^{d}$ & $6.81^{a-d}$ & $15.52^{f}$ & $1.95^{a-d}$ & $59.64^{e f}$ & $17.47^{h}$ \\
6 & $61.49^{a b}$ & $9.52^{a}$ & $17.06^{a}$ & $2.61^{a}$ & $71.02^{a b}$ & $19.68^{a}$ \\
7 & $62.91^{a}$ & $9.77^{a}$ & $16.81^{a-d}$ & $2.74^{a}$ & $72.67^{a}$ & $19.54^{a b}$ \\
8 & $57^{b-d}$ & $8.82^{a b}$ & $16.84^{a-c}$ & $2.48^{a-c}$ & $65.82^{b-d}$ & $19.32^{a-c}$ \\
9 & $59.68^{a-c}$ & $8.7^{a-c}$ & $16.84^{a-c}$ & $2.37^{a-d}$ & $68.38^{a-c}$ & $19.21^{a-d}$ \\
10 & $53.88^{d}$ & $5.79^{g h}$ & $15.89^{e f}$ & $1.62^{g-h}$ & $59.67^{e f}$ & $17.52^{h}$ \\
11 & $56.63^{b-d}$ & $8.49^{a-d}$ & $16.95^{a b}$ & $2.41^{a-c}$ & $65.12^{c d}$ & $19.36^{a-c}$ \\
12 & $54.51^{d}$ & $7.04^{a-d}$ & $16.92^{a b}$ & $1.98^{d-g}$ & $61.55^{d-f}$ & $18.9^{a-e}$ \\
13 & $53.41^{d}$ & $9.89^{a}$ & $16.6^{a-e}$ & $2.76^{a}$ & $63.3^{c-f}$ & $19.36^{a-c}$ \\
14 & $55.44^{c d}$ & $7.74^{b-e}$ & $16.35^{a-e}$ & $2.19^{b-e}$ & $63.18^{c-f}$ & $18.54^{c-f}$ \\
15 & $57.03^{b-d}$ & $5.39^{h}$ & $16.17^{b-f}$ & $1.39^{h}$ & $62.42^{d-f}$ & $17.56^{g h}$ \\
16 & $52.39^{d}$ & $7.09^{d-g}$ & $16.24^{b-f}$ & $2.09^{c-f}$ & $59.47^{f}$ & $18.33^{d-h}$ \\
17 & $55.32^{c d}$ & $7.29^{c-f}$ & $16.39^{a-e}$ & $2.09^{c-f}$ & $62.61^{d-f}$ & $18.48^{c-g}$ \\
PC & $55.79^{c d}$ & $6.49^{e-h}$ & $16.7^{a-d}$ & $1.86^{a-d}$ & $62.28^{d-f}$ & $18.56^{c-f}$ \\
T0 & $52.25^{d}$ & $5.87^{g h}$ & $15.83^{e f}$ & $1.68^{f-h}$ & $58.12^{f}$ & $17.51^{h}$ \\
CV (\%) & 7.31 & 14.73 & 3.82 & 15.3 & 6.7 & 3.96 \\
\hline
\end{tabular}

Letras diferentes dentro de cada columna indican diferencias significativas entre tratamientos (LSD, $\mathrm{p} \leq$ 0.05). EAS: Extracto de alga Sargassum spp.; PC: Producto comercial; T0: Control absoluto; CV: Coeficiente de variación; PFV: Peso fresco del vástago; PFR: Peso fresco de la raíz; PSV: Peso seco del vástago; PSR: Peso seco de la raíz; PFT: Peso fresco total; PST: Peso seco total.

Tabla 4. Efecto de los EAS sobre pigmentos fotosintéticos en hojas de plántulas de tomate.

\begin{tabular}{ccccc}
\hline $\begin{array}{c}\text { Tratamiento } \\
\text { (EAS) }\end{array}$ & $\begin{array}{c}\mathrm{Chl} \mathrm{a} \\
\left(\mathrm{mg} \mathrm{g}^{-1} \mathrm{PS}\right)\end{array}$ & $\begin{array}{c}\mathrm{Chl} \mathrm{b} \\
\left(\mathrm{mg} \mathrm{g}^{-1} \mathrm{PS}\right)\end{array}$ & $\begin{array}{c}\mathrm{Chl} \mathrm{T} \\
\left(\mathrm{mg} \mathrm{g}^{-1} \mathrm{PS}\right)\end{array}$ & $\begin{array}{c}\text { Caro } \\
\left(\mathrm{mg} \mathrm{g}^{-1} \mathrm{PS}\right)\end{array}$ \\
\hline 1 & $5.79^{b}$ & $2.91^{b}$ & $8.7^{b}$ & $0.22^{e-h}$ \\
2 & $5.2^{f}$ & $2.62^{e f}$ & $7.82^{e f}$ & $0.31^{1-d}$ \\
3 & $4.86^{i}$ & $2.54^{e f}$ & $7.4^{i j}$ & $0.21^{e-h}$ \\
4 & $5.14^{f}$ & $2.61^{e f}$ & $7.75^{f}$ & $0.22^{e-h}$ \\
5 & $5.54^{c}$ & $2.82^{b-d}$ & $8.36^{c}$ & $0.24^{d-g}$ \\
6 & $4.83^{i}$ & $2.56^{e f}$ & $7.39^{j}$ & $0.13^{i}$ \\
7 & $5.84^{b}$ & $2.87^{b}$ & $8.71^{b}$ & $0.25^{c-f}$ \\
8 & $4.87^{h i}$ & $2.56^{e f}$ & $7.43^{h-j}$ & $0.19^{f-i}$ \\
9 & $4.95^{g h}$ & $2.61^{e f}$ & $7.56^{g-i}$ & $0.15^{h i}$ \\
10 & $5.31^{e}$ & $2.68^{c-e}$ & $7.99^{d e}$ & $0.22^{e-h}$ \\
11 & $4.91^{h i}$ & $2.56^{e f}$ & $7.47^{h-j}$ & $0.15^{h i}$ \\
12 & $6.23^{a}$ & $3.11^{a}$ & $9.34^{a}$ & $0.34^{a b}$ \\
13 & $5.03^{g}$ & $2.66^{d e}$ & $7.69^{f g}$ & $0.2^{f-i}$ \\
14 & $5.44^{d}$ & $2.83^{b c}$ & $8.27^{c}$ & $0.33^{b}$ \\
15 & $5.76^{b}$ & $2.93^{b}$ & $8.69^{b}$ & $0.32^{b c}$ \\
16 & $5.6^{c}$ & $2.83^{b-d}$ & $8.43^{c}$ & $0.41^{a}$ \\
17 & $4.99^{g}$ & $2.58^{e f}$ & $7.57^{g h}$ & $0.24^{d-g}$ \\
PC & $5.36^{e}$ & $2.67^{c-e}$ & $8.03^{d}$ & $0.29^{b-e}$ \\
T0 & $4.66^{j}$ & $2.47^{f}$ & $7.12^{k}$ & $0.17^{g-i}$ \\
CV (\%) & 0.94 & 3.7 & 1.27 & 19.63 \\
\hline
\end{tabular}

Letras diferentes dentro de cada columna indican diferencias significativas entre tratamientos (LSD, $\mathrm{p} \leq 0.05$ ). EAS: Extracto de alga Sargassum spp.; PC: Producto comercial; T0: Control absoluto; CV: Coeficiente de variación; Chl a: Clorofila a; Chl b: Clorofila b; Chl T: Clorofila total; Caro: Carotenoides; PS: Peso seco. 

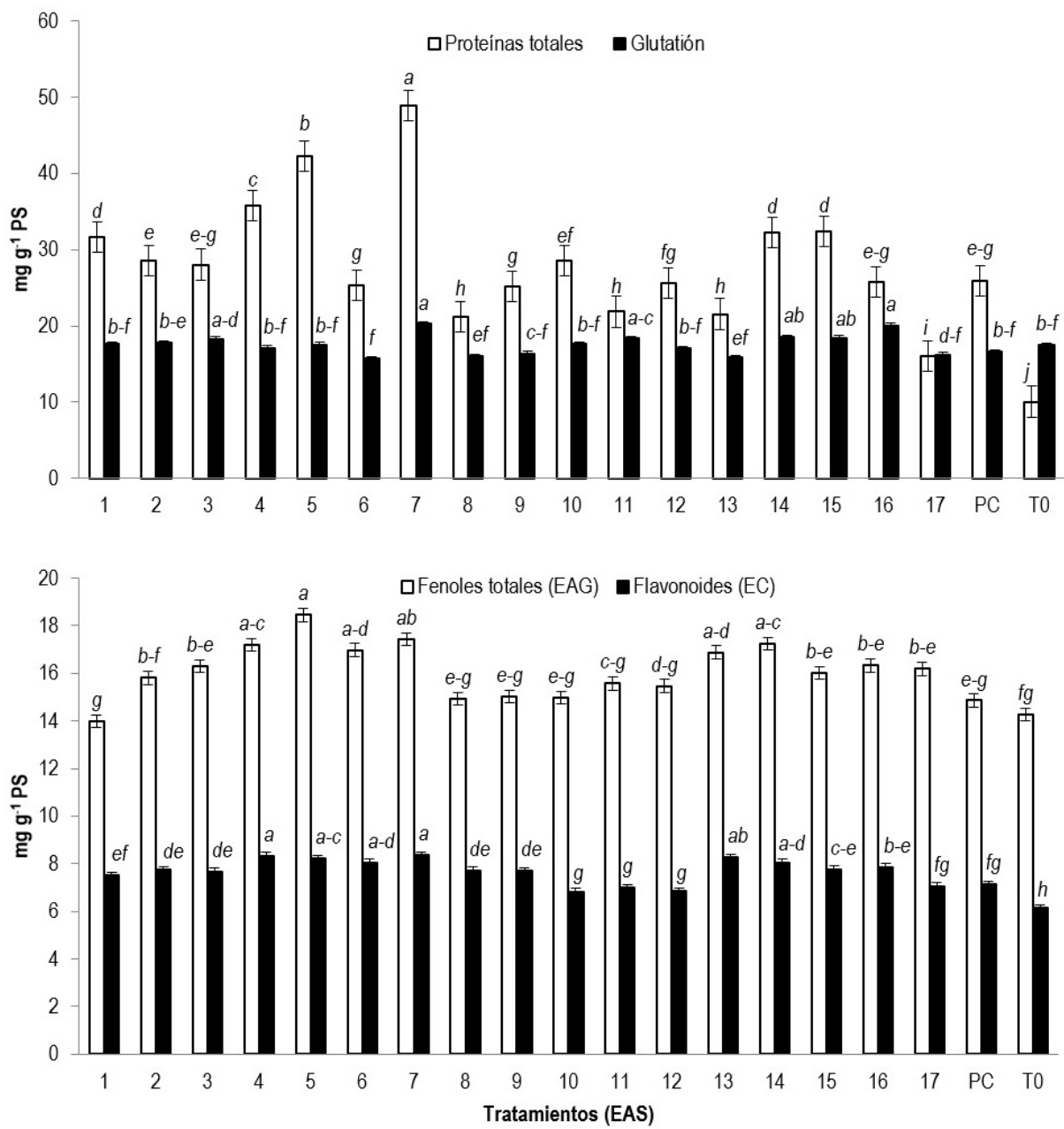

Figura 1. Proteínas y compuestos antioxidantes en hojas de plántulas de tomate tratadas con aplicaciones foliares de EAS. Letras diferentes indican diferencias significativas entre tratamientos (LSD, $p \leq$ 0.05). PS: Peso seco; PC: Producto comercial; T0: Control absoluto; EAG: Equivalentes de ácido gálico; EC: Equivalentes de catequina; EAS: Extracto de alga Sargassum spp. Los intervalos de las barras representan el error estándar.

nos indica que entre más severa fue la condición de extracción $\left(160^{\circ} \mathrm{C} / 30 \mathrm{~min} / 50 \%\right.$ etanol), la obtención de metabolitos fue mayor. En este sentido al elevar la temperatura del solvente (agua: etanol) la extracción es mayor, ya que, se facilita la lisis celular, provocando una mayor liberación de compuestos bioactivos, y el mantener dichas condiciones por lapsos más prolongados aumenta el rendimiento de la extracción (Heffernan et al. 2016, Casagrande et al. 2018). Es por eso que la mayor concentración de proteínas, aminoácidos y glutatión lo presentó el tratamiento EAS7, el cual, presenta las condiciones más elevadas de extracción (Tabla 1). Estos resultados son similares a los reportados por Ferreira et al. (2019) quienes muestran que en Fucus vesiculosus a temperaturas y tiempos más elevados $\left(120{ }^{\circ} \mathrm{C}\right.$ y $4 \mathrm{~h}$, respectivamente) de extracción se obtienen las mejores concentraciones de compuestos bioactivos. De la misma manera Fu et al. (2016) muestran un aumento en la capacidad antioxidante de extractos de diferentes algas en la medida que aumenta el tiempo de extracción.

Hay estudios que indican que a mayores temperaturas y tiempos de extracción es posible obtener 


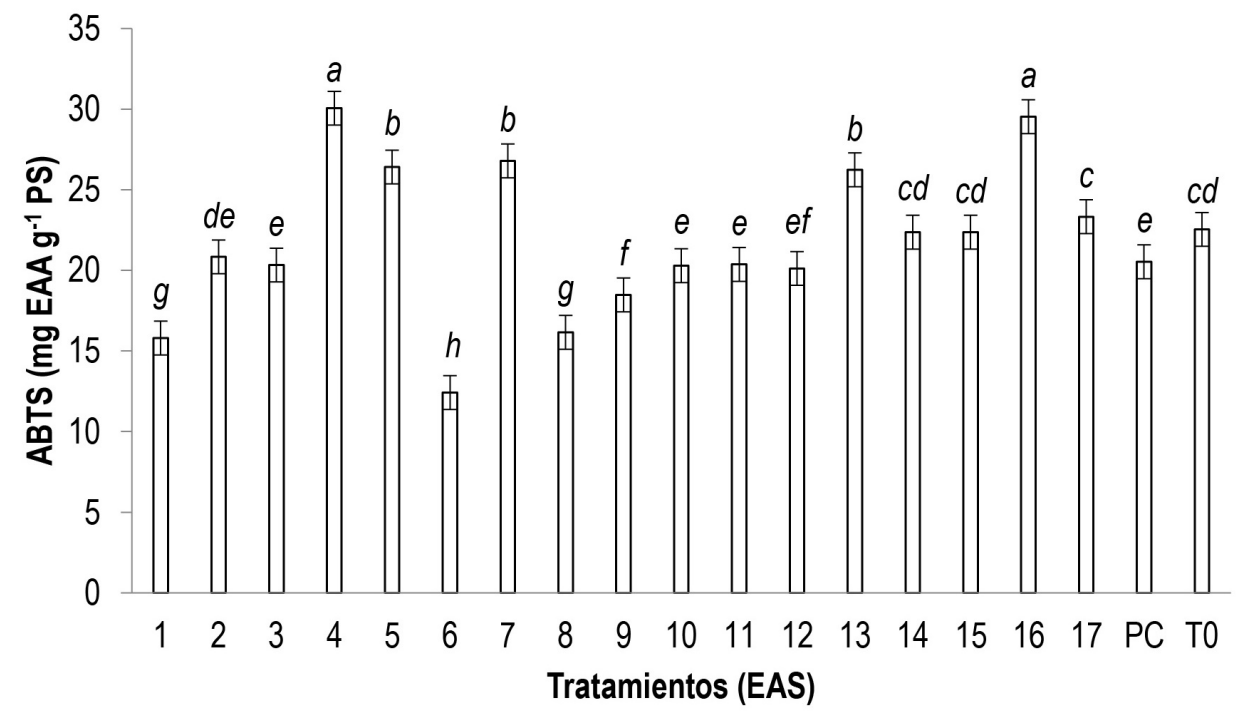

Figura 2. Capacidad antioxidante hidrofílica en hojas de plántulas de tomate tratadas con aplicaciones foliares de EAS. Letras diferentes indican diferencias significativas entre tratamientos (LSD, $p \leq 0.05$ ). ABTS: 2,2'-Azino-bis (3-ethylbenzothiazoline-6-sulfonic acid); EAA: Equivalentes de ácido ascórbico; PS: Peso seco; PC: Producto comercial; T0: Control absoluto; EAS: Extracto de alga Sargassum spp. Los intervalos de las barras representan el error estándar.

mayores concentraciones de compuestos, pero a su vez, las mismas condiciones pueden ocasionar la degradación de los mismos (Ferreira et al. 2019). Lo cual, aparentemente no sucedió con los compuestos que se determinaron en este estudio, posiblemente porque se necesitan condiciones más severas para su degradación, ya que, los enlaces que presentan estos compuestos (proteínas, aminoácidos y glutatión) son covalentes, los cuales, son los más fuertes y difíciles de romper (Lin et al. 2020).

\section{Parámetros de crecimiento de las plántulas}

La obtención de plántulas de calidad depende de su genética, fisiología y un ambiente favorable para el desarrollo de raíz y vástago (Giménez et al. 2008). En la actualidad la producción y propagación de plántulas está afectada por diferentes estrés ambientales que deterioran su calidad, lo que, las hace propensas a infecciones por patógenos (Ruan et al. 2009), lo que lleva a buscar alternativas. Los resultados obtenidos, posiblemente son atribuidos a la mayor concentración de metabolitos nitrogenados en el EAS7 (proteínas, aminoácidos y glutatión), los cuales, al ser fuentes de $\mathrm{N}$ y precursores hormonales modifican positivamente el metabolismo, lo que mejora el crecimiento y la acumulación de biomasa en los cultivos (Hernández et al. 2009, Hernández-Herrera et al. 2014, Teixeira et al. 2017).

La producción de plántulas sanas y vigorosas favorecen su arraigo y buen desarrollo en campo (Preciado-Rangel et al. 2002). Los resultados son consistentes con los reportados por Kumari et al. (2011) quienes demostraron que la aplicación foliar, en drench y combinada de extractos de Sargassum johnstonii mejoró la altura, longitud de los brotes, numero de hojas y la longitud de la raíz en plantas de tomate a dosis de $10 \%$. Lo que se atribuye a la presencia de auxinas, citoquininas y nutrientes esenciales en los extractos utilizados. Sobre lo mismo Kalaivanan y Venkatesalu (2012) evaluaron el efecto de la aplicación de extractos de Sargassum myriocystum como estimulante en plántulas de frijol, reportando que dosis bajas (10\%) incrementan la altura, longitud de la raíz, peso fresco y seco de las plántulas; mientras que dosis altas, mayores de 10\%, mostraron tendencias decrecientes. Mientras que Vijayanand et al. (2014) al evaluar aspersiones foliares de extractos de Sargassum wightii en el cultivo de frijol indican que tratamientos a dosis de $1.5 \%$, al igual que este experimento mejoraron los parámetros de 
crecimiento del cultivo en comparación con el control.

\section{Pigmentos fotosintéticos}

La capacidad fotosintética de las hojas está relacionada con el contenido de $\mathrm{N}$, principalmente porque las proteínas de los tilacoides y las que actúan en el ciclo de Calvin representan la mayoría del $\mathrm{N}$ foliar (Latsague et al. 2014, Sánchez et al. 2018), lo cual, está relacionado con la concentración de metabolitos nitrogenados en los EAS, motivo por el cual, se cree que todos los EAS incluido el producto comercial incrementaron la concentración de pigmentos en comparación al control absoluto.

También hay estudios que demuestran que los extractos de algas pardas contienen betaínas, compuesto que cumple diversas funciones en las plantas, como osmoprotector ante el déficit hídrico, sin embargo, su rol principal está en el incremento de la actividad fotosintética, ya que, evita la degradación de los pigmentos (Blunden et al. 1996, Estaji et al. 2019). Como lo mencionan Zermeño-González et al. (2015) quienes demuestran que la adición foliar y drench de extractos de Sargassum spp. a dosis de $1 \mathrm{~L} \mathrm{ha}^{-1}$ en vid aumentó significativamente la concentración de clorofilas en las hojas. Mientras que Vijayanand et al. (2014) también indican que aspersiones foliares de extractos de Sargassum wightii a dosis de $1.5 \%$ incrementan la concentración de pigmentos fotosintéticos en hojas de frijol.

\section{Proteínas y estado antioxidante de las plántulas}

Un antioxidante es cualquier molécula que retrasa, previene o elimina el daño oxidativo a otras moléculas, con la eliminación o neutralización de especies reactivas de oxígeno, nitrógeno y azufre producidas durante el estrés, las cuales, son causantes de dicho daño oxidativo, induciendo así tolerancia en las plantas (Olson et al. 2017, Kapoor et al. 2019, Chib et al. 2020). Los resultados de esta investigación posiblemente son producto de la mayor concentración de metabolitos en el EAS7 (Tabla 1), los cuales, actúan como bioestimulantes, como los compuestos nitrogenados presentes (proteínas, aminoácidos y glutatión). Al respecto, varios estudios demuestran que este tipo de metabolitos tienen la ca- pacidad de activar el sistema de defensa de las plantas, al inducir mayor síntesis de compuestos antioxidantes, como lo indican Kollist et al. (2019) y Shukla et al. (2019) quienes reportan que las membranas celulares cuentan con una serie de sensores, los cuales, tienen la función de unirse al metabolito y eventualmente desencadenar una serie de señalizaciones celulares que resultan en la activación del sistema de defensa, por lo cual, se incrementa la síntesis y actividad de compuestos antioxidantes, como los enzimáticos (superóxido dismutasa, catalasa, ascorbato peroxidasa y glutatión peroxidasa) y no enzimáticos (fenoles, carotenoides, glutatión y vitaminas) (He et al. 2018, Shukla et al. 2019). Los antioxidantes enzimáticos se consideran la primera línea de defensa, la cual dificulta la producción de radicales libres que conduce al estrés oxidativo, en cambio los antioxidantes no enzimáticos participan en la segunda línea de defensa, que se encarga de inhibir la producción de daños causados por la reacción oxidativa (Haida y Hakiman 2019).

Existen estudios que indican que los EAS pueden incrementar la síntesis de compuestos antioxidantes, como lo muestran Vijayanand et al. (2014) quienes afirman que la aplicación foliar de extractos de Sargassum wightii a dosis de $1.5 \%$ aumentan la concentración de azúcares reductores y ácido ascórbico en las hojas de frijol. Mientras que Kumari et al. (2011) al evaluar la aplicación foliar, drench y combinada de extractos de Sargassum johnstonii sobre el contenido de compuestos bioactivos en frutos y hojas de tomate, reportan que todas las formas de aplicación mejoraron la síntesis de proteínas, azúcares, fenoles, ácido ascórbico y licopeno a dosis de $10 \%$. Mientras que Vasantharaja et al. (2019) demostraron que la adición foliar de extractos Sargassum swartzii al 3\% incrementó la concentración de proteínas, fenoles totales, flavonoides y capacidad antioxidante DPPH en frijol. De forma similar Kasim et al. (2015) informaron que el pretratamiento de semillas de trigo con extractos de Sargassum latifolium al $1.5 \%$ mejoró el mecanismo de defensa (enzimático y no enzimático) de las plántulas al ser estresadas por sequía.

Es importante mencionar que los EAS tam- 
bién son ricos en carotenoides, vitaminas, fenoles, hormonas, azucares y minerales, los cuales, posiblemente también influyeron en todos los resultados y no solo los nitrogenados. Aunado a esto se puede observar que existen respuestas muy diferentes en cada una de las variables estudiadas de acuerdo al tratamiento aplicado, lo cual, depende del tipo de material, sistema de producción, condiciones ambientales y la concentración de los EAS. Cada uno de estos factores afecta de manera diferente el desarrollo y síntesis de metabolitos en las plantas. El material vegetal y el sistema de producción son de importancia, ya que, de esta manera se pueden controlar ciertos factores ambientales que son causantes de estrés (Holzkämper 2017, Khaki et al. 2019). La concentración de los EAS es algo que siempre se tiene que tomar en cuenta, y lo más recomendable de acuerdo a diferentes investigaciones es aplicar este tipo de bioestimulantes a dosis bajas (Kauffman et al. 2007).

\section{CONCLUSIONES}

Los resultados indican que los EAS modificaron de forma positiva los parámetros de crecimiento y de compuestos antioxidantes en las plántulas de tomate. El EAS7 fue el que mejores resultados presentó al aumentar el crecimiento, biomasa y la concentración de compuestos antioxidantes. En cuanto a los pigmentos fotosintéticos el EAS12 fue el que mejores resultados ocasionó. Lo que indica que los EAS son una opción viable para producir plántulas más vigorosas y con un mejor sistema de defensa, lo cual, mejora la adaptación de las plántulas después del trasplante. En cuanto a la caracterización de los EAS, el EAS7 $\left(160{ }^{\circ} \mathrm{C} / 30 \mathrm{~min} / 50 \%\right.$ de etanol) fue el que presentó la mayor concentración de metabolitos nitrogenados, esto posiblemente a que las condiciones de extracción fueron las más severas, por lo cual, se cree que provocó los mejores efectos en las plántulas. Se recomienda el uso del EAS7, en estos sistemas de producción.

\section{AGRADECIMIENTOS}

Este trabajo fue realizado gracias al Consejo Nacional de Ciencia y Tecnología (CONACyT). Número de CVU 818215. Doctorado en Ciencias en Agricultura Protegida, Universidad Autónoma Agraria Antonio Narro. Saltillo, México.

\section{LITERATURA CITADA}

Abbas M, Anwar J, Zafar-Ul-Hye M, Khan RI, Saleem M, Rahi AA, Danish S, Datta R (2020) Effect of seaweed extract on productivity and quality attributes of four onion cultivars. Horticulturae 6: 28. DOI: 10.3390/horticulturae6020028.

Bai Y, Sunarti S, Kissoudis C, Visser RG, Van Der Linden CG (2018) The role of tomato WRKY genes in plant responses to combined abiotic and biotic stresses. Frontiers in Plant Science 9: 801. DOI: 10.3389/fpls.2018. 00801.

Blunden G, Jenkins T, Liu YW (1996) Enhanced leaf chlorophyll levels in plants treated with seaweed extract. Journal of Applied Phycology 8: 535-543.

Bradford MM (1976) A rapid and sensitive method for the quantitation of protein utilizing the principle of proteindye binding. Analytical Biochemistry 72: 248-254.

Brown EM, Allsopp PJ, Magee PJ, Gill Cl, Nitecki S, Strain CR, McSorley EM (2014) Seaweed and human health. Nutrition Reviews 72: 205-216

Casagrande M, Zanela J, Júnior AW, Busso C, Wouk J, lurckevicz G, Montanher PF, Yamashita F, Malfatti CRM (2018) Influence of time, temperature and solvent on the extraction of bioactive compounds of Baccharis 
dracunculifolia: In vitro antioxidant activity, antimicrobial potential, and phenolic compound quantification. Industrial Crops and products 125: 207-219.

Chib A, Gupta N, Bhat A, Anjum N, Yadav G (2020) Role of antioxidants in food. International Journal Chemical Studies 8: 2354-2361.

du Jardin P (2015) Plant biostimulants: Definition, concept, main categories and regulation. Scientia Horticulturae 196: 3-14.

Ertani A, Francioso O, Tinti A, Schiavon M, Pizzeghello D, Nardi S (2018) Evaluation of seaweed extracts from Laminaria and Ascophyllum nodosum spp. As biostimulants in Zea mays L. using a combination of chemical, biochemical and morphological approaches. Frontiers in Plant Science 9: 428. DOI: 10.3389/fpls.2018. 00428.

Estaji A, Kalaji HM, Karimi HR, Roosta HR, Moosavi-Nezhad SM (2019) How glycine betaine induces tolerance of cucumber plants to salinity stress. Photosynthetica 57: 753-761.

Ferreira RM, Ramalho-Ribeiro A, Patinha C, Silva A, Cardoso SM, Costa R (2019) Water extraction kinetics of bioactive compounds of Fucus vesiculosus. Molecules 24: 3408. DOI: 10.3390/molecules24183408.

Fu CWF, Ho CW, Yong WTL, Abas F, Tan TB, Tan CP (2016) Extraction of phenolic antioxidants from four selected seaweeds obtained from Sabah. International Food Research Journal 23: 2363-2369.

Giménez G, Andriolo JL, Janisch D, Godoi R (2008) Closed soilless growing system for producing strawberry bare root transplants and runner tips. Pesquisa Agropecuária Brasileira 43: 1757-1761.

González-García Y, López-Vargas ER, Cadenas-Pliego G, Benavides-Mendoza A, González-Morales S, RobledoOlivo A, Alpuche-Solís AG, Juárez-Maldonado A (2019) Impact of carbon nanomaterials on the antioxidant system of tomato seedlings. International Journal of Molecular Sciences 20: 5858. DOI: 10.3390/ijms20235 858.

Haida Z, Hakiman M (2019) A comprehensive review on the determination of enzymatic assay and nonenzymatic antioxidant activities. Food Science \& Nutrition 7: 1555-1563.

He Y, Zhou J, Shan L, Meng X (2018) Plant cell surface receptor-mediated signaling-a common theme amid diversity. Journal of Cell Science 131: 1-11.

Heffernan N, Smyth TJ, FitzGerald RJ, Vila-Soler A, Mendiola J, Ibáñez E, Brunton NP (2016) Comparison of extraction methods for selected carotenoids from macroalgae and the assessment of their seasonal/spatial variation. Innovative Food Science \& Emerging Technologies 37: 221-228.

Hernández MI, Salgado JM, Chailloux M, Moreno V, Mojena M (2009) Relaciones nitrógeno-potasio en fertirriego para el cultivo protegido del tomate (Solanum lycopersicum L.) y su efecto en la acumulación de biomasa y extracción de nutrientes. Cultivos Tropicales 30: 71-78.

Hernández-Herrera RM, Santacruz-Ruvalcaba F, Ruiz-López MA, Norrie J, Hernández-Carmona G (2014) Effect of liquid seaweed extracts on growth of tomato seedlings (Solanum lycopersicum L.). Journal of Applied Phycology 26: 619-628.

Holzkämper A (2017) Adapting agricultural production systems to climate change-what's the use of models? Agriculture 7: 86. DOI: 10.3390/agriculture7100086.

Jayaraman J, Norrie J, Punja ZK (2011) Commercial extract from the brown seaweed Ascophyllum nodosum reduces fungal diseases in greenhouse cucumber. Journal of Applied Phycology 23: 353-361. 
Kalaivanan C, Venkatesalu V (2012) Utilization of seaweed Sargassum myriocystum extracts as a stimulant of seedlings of Vigna mungo (L.) Hepper. Spanish Journal of Agricultural Research 10: 466-470.

Kapoor D, Singh S, Kumar V, Romero R, Prasad R, Singh J (2019) Antioxidant enzymes regulation in plants in reference to reactive oxygen species (ROS) and reactive nitrogen species (RNS). Plant Gene 19: 100182. DOI: 10.1016/j.plgene.2019.100182.

Kasim WA, Hamada EA, El-Din NGS, Eskander S (2015) Influence of seaweed extracts on the growth, some metabolic activities and yield of wheat grown under drought stress. International Journal of Agronomy and Agricultural Research 7: 173-189.

Kauffman GL, Kneivel DP, Watschke TL (2007) Effects of a biostimulant on the heat tolerance associated with photosynthetic capacity, membrane thermostability, and polyphenol production of perennial ryegrass. Crop Science 47: 261-267.

Khaki S, Khalilzadeh Z, Wang L (2019) Classification of crop tolerance to heat and drought-a deep convolutional neural networks approach. Agronomy 9: 833. DOI: 10.3390/agronomy9120833.

Kollist H, Zandalinas SI, Sengupta S, Nuhkat M, Kangasjärvi J, Mittler R (2019) Rapid responses to abiotic stress: Priming the landscape for the signal transduction network. Trends in Plant Science 24: 25-37.

Kumari R, Kaur I, Bhatnagar AK (2011) Effect of aqueous extract of Sargassum johnstonii Setchell \& Gardner on growth, yield and quality of Lycopersicon esculentum Mill. Journal of Applied Phycology 23: 623-633.

Latsague M, Sáez P, Mora M (2014) Efecto de la fertilización con nitrógeno, fósforo y potasio, sobre el contenido foliar de carbohidratos, proteínas y pigmentos fotosintéticos en plantas de Berberidopsis corallina Hookf. Gayana Botánica 71: 37-42.

Lin X, Wang J, Ding B, Ma X, Tian H (2020) Tunable-Emission Amorphous Room-Temperature Phosphorescent Polymers Based on Thermoreversible Dynamic Covalent Bonds. Angewandte Chemie 133: 3501-3505.

Michalak I, Chojnacka K (2014) Algal extracts: Technology and advances. Engineering in Life Sciences 14: 581-591.

Michalak I, Chojnacka K (2015) Algae as production systems of bioactive compounds. Engineering in Life Sciences 15: 160-176.

N'Yeurt ADR, Lese V (2015) The proliferating brown alga Sargassum polycystum in Tuvalu, South Pacific: assessment of the bloom and applications to local agriculture and sustainable energy. Journal of Applied Phycology 27: 2037-2045.

Olson KR, Gao Y, DeLeon ER, Arif M, Arif F, Arora N, Straub KD (2017) Catalase as a sulfide-sulfur oxidoreductase: an ancient (and modern?) regulator of reactive sulfur species (RSS). Redox Biology 12: 325339.

Perez CMT, Pajares IG, Alcantara VA, Simbahan JF (2018) Bacterial laminarinase for application in ethanol production from brown algae Sargassum sp. using halotolerant yeast. Biofuel Research Journal 5: 792797.

Preciado-Rangel P, Baca-Castillo GA, Tirado-Torres JL, Kohashi-Shibata J, Tijerina-Chávez L, Martínez-Garza A (2002) Nitrógeno y potasio en la producción de plántulas de melón. Terra Latinoamericana 20: 267-276.

Ramya SS, Nagaraj S, Vijayanand N (2011) Influence of Seaweed Liquid Extracts on Growth, Biochemical and Yield Characteristics of Cyamopsis tetragonolaba (L.) Taub. Journal of Phytology 3: 37-41. 
Ramya SS, Vijayanand N, Rathinavel S (2015) Foliar application of liquid biofertilizer of brown alga Stoechospermum marginatum on growth, biochemical and yield of Solanum melongena. International Journal of Recycling of Organic Waste in Agriculture 4: 167-173.

Re R, Pellegrini N, Proteggente A, Pannala A, Yang M, Rice-Evans C (1999) Antioxidant activity applying an improved ABTS radical cation decolorization assay. Free Radical Biology and Medicine 26: 1231-1237.

Ruan J, Yoon C, Yeoung Y, Larson KD, Ponce L (2009) Efficacy of highland production of strawberry transplants. African Journal of Biotechnology 8: 1497-1501.

Salehi B, Sharifi-Rad R, Sharopov F, Namiesnik J, Roointan A, Kamle M (2019) Beneficial effects and potential risks of tomato consumption for human health: An overview. Nutrition 62: 201-208.

Sánchez E, Ruiz JM, Romero L, Preciado-Rangel P, Flores-Córdova MA, Márquez-Quiroz C (2018) ¿Son los pigmentos fotosintéticos buenos indicadores de la relación del nitrógeno, fósforo y potasio en frijol ejotero? Ecosistemas y Recursos Agropecuarios 5: 387-398.

Shukla PS, Mantin EG, Adil M, Bajpai S, Critchley AT, Prithiviraj B (2019) Ascophyllum nodosum-based biostimulants: Sustainable applications in agriculture for the stimulation of plant growth, stress tolerance, and disease management. Frontiers in Plant Science 10: 655. DOI: 10.3389/fpls.2019.00655.

Singleton VL, Orthofer R, Lamuela-Raventos RM (1999) Analisys of total phenols and other oxidation sobstrates and antioxidants by means of Folin Ciocalteau reagent. Methods in Enzymology 299: 152-178.

Steiner AA (1961) A universal method for preparing nutrient solutions of a certain desired composition. Plant and Soil 15: 134-154.

Teixeira WF, Fagan EB, Soares LH, Umburanas RC, Reichardt K, Neto DD (2017) Foliar and seed application of amino acids affects the antioxidant metabolism of the soybean crop. Frontiers in Plant Science 8: 327. DOI: 10.3389/fpls.2017.00327.

Trejo-Valencia R, Sánchez-Acosta L, Fortis-Hernández M, Preciado-Rangel P, Gallegos-Robles MÁ, AntonioCruz RDC, Vázquez-Vázquez C (2018) Effect of Seaweed Aqueous Extracts and Compost on Vegetative Growth, Yield, and Nutraceutical Quality of Cucumber (Cucumis sativus L.) Fruit. Agronomy 8: 264. DOI: 10.3390/agronomy8110264.

Vasantharaja R, Abraham LS, Inbakandan D, Thirugnanasambandam R, Senthilvelan T, Jabeen SA, Prakash P (2019) Influence of seaweed extracts on growth, phytochemical contents and antioxidant capacity of cowpea (Vigna unguiculata L. Walp). Biocatalysis and Agricultural Biotechnology 17: 589-594.

Vijayanand N, Ramya SS, Rathinavel S (2014) Potential of liquid extracts of Sargassum wightii on growth, biochemical and yield parameters of cluster bean plant. Asian Pacific Journal of Reproduction 3: 150-155.

Wellburn AR (1994) The spectral determination of chlorophylls $a$ and $b$, as well as total carotenoids, using various solvents with spectrophotometers of different resolution. Journal of Plant Physiology 144: 307-313.

Xue T, Hartikainen H, Piironen V (2001) Antioxidative and growth-promoting effect of selenium on senescing lettuce. Plant and Soil 237: 55-61.

Yemm EW, Cocking EC (1955) The determination of amino-acids with ninhydrin. Analyst 80: 209-214.

Zermeño-González A, López-Rodríguez BR, Melendres-Alvarez Al, Ramírez-Rodríguez H, Cárdenas-Palomo JO, Munguía-López JP (2015) Extracto de alga marina y su relación con fotosíntesis y rendimiento de una plantación de vid. Revista Mexicana de Ciencias Agrícolas 6: 2437-2446. 
Zhishen J, Mengcheng T, Jianming W (1999) The determination of flavonoid contents in mulberry and their scavenging effects on superoxide radicals. Food Chemistry 64: 555-559. 